\title{
New directions in pharmaceutical amorphous materials and amorphous solid dispersions, a tribute to Professor George Zografi - Proceedings of the June 2016 Land O'Lakes Conference
}

\author{
Ann Newman ${ }^{1 *}$, Jayne E. Hastedt ${ }^{2}$ and Mehran Yazdanian ${ }^{3}$
}

\begin{abstract}
The University of Wisconsin-Madison June Land O'Lakes Conference on Research and Development is held every year and is recognized worldwide as a premier teaching conference for pharmaceutical scientists. The conference held in June 2016 was a tribute to the ground-breaking work of Emeritus Professor and Dean George Zografi of School of Pharmacy, University of Wisconsin-Madison. This paper provides a summary of the wide range of topics in the areas of amorphous drugs, amorphous solid dispersions, mesophases, mesoporous supports, cocrystals, and related themes that were covered at this conference.
\end{abstract}

Keywords: Amorphous, Amorphous solid dispersions, Amorphous structure, Mesoporous supports, Mesophases, Co-crystals, Process induced transformations

\section{Introduction}

The University of Wisconsin-Madison June Land O'Lakes Conference on Research and Development is held every year and is recognized worldwide as one of the premier teaching conferences for pharmaceutical scientists who want to learn about the latest trends and hot topics in formulation. The conference held in June 2016 was a tribute to the ground-breaking work of Emeritus Professor and Dean George Zografi of the School of Pharmacy, University of Wisconsin-Madison. This paper provides a summary of the wide range of topics in the areas of amorphous drugs, amorphous solid dispersions (ASDs), and related themes that were covered at this conference.

Professor George Zografi received a B.S. in Pharmacy from Columbia University and M.S. and Ph.D. in Pharmaceutics from the University of Michigan. He started his academic career on the College of Pharmacy faculties of Columbia University (1960-1964) and the

\footnotetext{
* Correspondence: ann.newman@seventhstreetdev.com

'Seventh Street Development Group LLC, Kure Beach, NC 28449, USA Full list of author information is available at the end of the article
}

University of Michigan (1964-1972) before joining the University of Wisconsin-Madison in 1972. He served as Dean of the School of Pharmacy From 1975 to 1980 and was named the Edward Kremers Professor of Pharmaceutical Sciences in 1997. Professor Zografi's research interests have been focused in three scientific areas related to drug product development: the physical chemical properties of amorphous solids and amorphous solid dispersions; the hygroscopic properties of solids and their impact on physical and chemical instabilities; and the surface chemistry of lipids, polymers, and proteins in monolayer and bilayer systems (Fig. 1). Professor Zografi's long and distinguished career included mentoring 26 Ph.D. and 20 M.S. students as well 18 post-doctoral fellows. He has published 157 papers and 21 book chapters and reviews along with 90 invited lectures and 116 invited industrial seminars. He officially retired from the University of Wisconsin-Madison in 2006, but continues to inspire scientists throughout the industry with papers and presentations.

The work from Professor Zografi's laboratory has shaped countless scientists and projects over the years, 


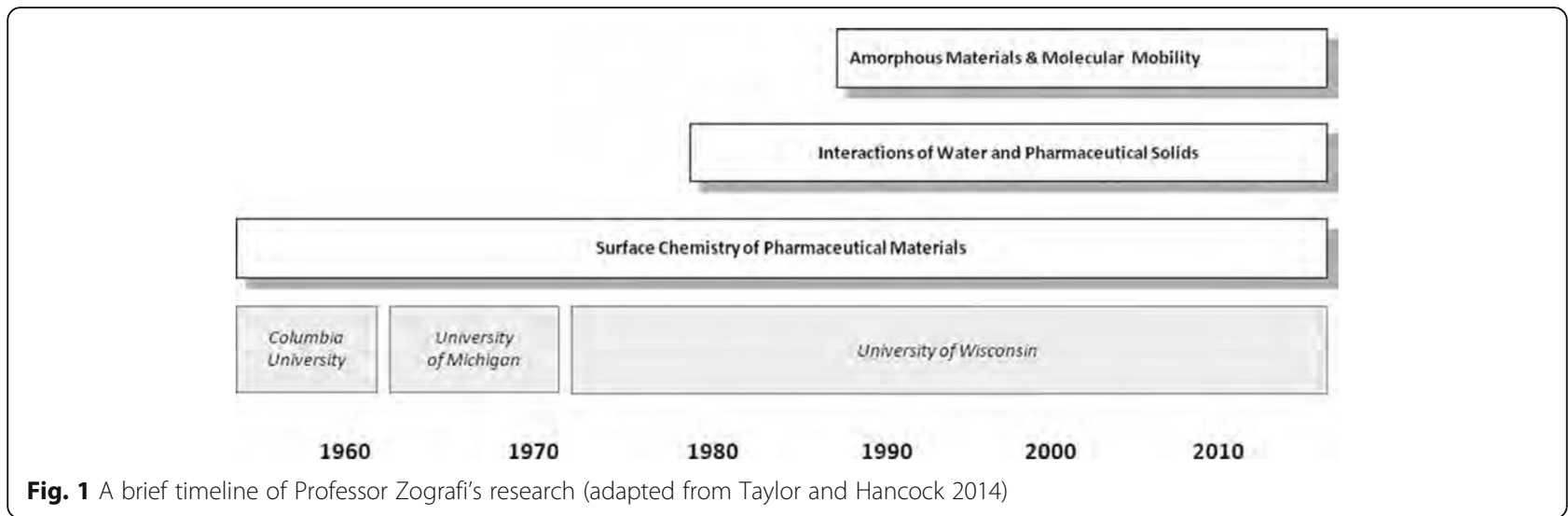

as evidenced by the wide variety of talks and topics at this Land'O'Lakes Conference. New and innovative areas of amorphous drug substance/amorphous solid dispersion understanding and development that have been built on his research were explored. Key topics included characterization, stability, computational studies, amorphous structure, and process induced changes. Related areas, such as mesophases and mesoporous supports, were also discussed. The conference finished with a regulatory discussion on new Biopharmaceutical Classification System (BCS) and co-crystal guidances with speakers from the Food and Drug Administration (FDA) and industry.

This paper surveys the diverse topics presented at the conference that were influenced by Professor Zografi's work on amorphous materials. There are many other areas that were not included in the conference, such as his work with surface chemistry and interactions with water, that continue to shape the development of pharmaceutical compounds.

\section{Academic and industrial perspectives of amorphous materials}

An academic perspective on amorphous materials was presented by Professor Lian $\mathrm{Yu}$, University of Wisconsin-Madison, focusing on the physical stability of amorphous solids and the central role for surface mobility. Molecular glasses are produced by freezing liquid structures. Some advantages to glasses are spatial uniformity over long distances, incorporation of multiple components, and increased solubility when compared to crystals. Disadvantages include crystallization and physical aging. Many models for crystal growth have been reported since 1900, with the latest outlining growth on surface fractures and highlighting surface mobility (Powell et al. 2015). It has been found that surface diffusion $\left(D_{s}\right)$ is the lateral translation of surface particles and not vertical mixing of the bulk diffusion $\left(D_{v}\right)$ into the core of the solid. For five molecular glasses it was found that $D_{s}$ decreased with increasing molecular size and hydrogen bonding. Experiments have shown that glasses exhibiting van der Waals forces (vdW glasses) exhibit faster surface diffusion than glasses with significant hydrogen bonding (network glasses). Surface mobility was found to have a central role in the stability of molecular glasses, with surface crystal growth occurring laterally and upward, not down into the bulk (Hasebe et al. 2015). Glasses with faster surface diffusion result in faster crystal growth, and result in depletion zones on the surface (Fig. 2). Fast bulk crystallization was found to occur along fractures in the glass; many pharmaceutical glasses are easy to fracture based on fracture toughness modulus (Powell et al. 2015). An example with indomethacin shows that a glass with no fractures shows only a glass transition $\left(\mathrm{T}_{\mathrm{g}}\right)$ by differential scanning calorimetry (DSC), whereas a fractured glass exhibits a crystallization exotherm in the DSC curve (Ayenew et al. 2012) indicating that fracture can cause fast crystal growth along cracks and at the same rate as on free surfaces. When estimating the stability of glasses, surface mobility must be considered, as well as the amount of fractures present that may increase bulk crystallization. Stability could be improved with "dry" surface coatings or mesoporous carriers. This body of work showed that understanding the mechanisms for crystallization and, ultimately, amorphous stability, is still an active area of research. As these new areas are further investigated, development of amorphous materials and amorphous dispersions may be developed differently based on a new understanding of these systems.

An industrial perspective on amorphous materials and amorphous solid dispersions was presented by Dr. Mike Hageman, Bristol-Myers Squibb. Increased chemical diversity of an active pharmaceutical ingredient (API) requires a more integrated role of drug delivery during lead optimization (Fig. 3). Integrating ASDs into drug discovery offers several advantages and potential disadvantages, as outlined in Table 1. Once an ASD is used in discovery, it should be assessed for possible use later in 

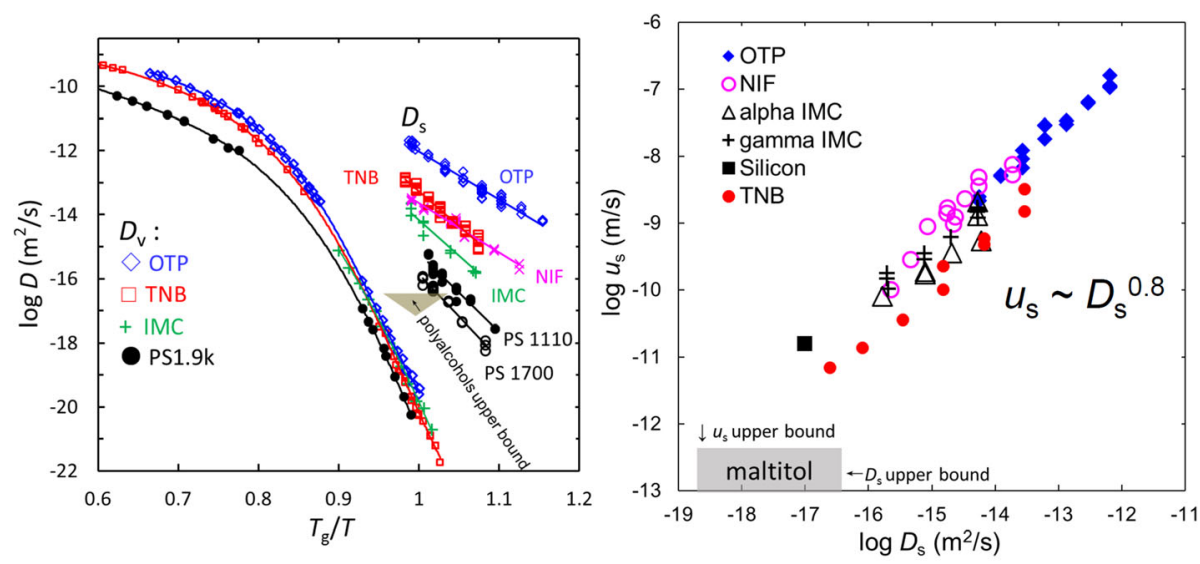

\section{$u_{\mathrm{s}}$ of maltitol too slow to measure}

Fig. 2 Left: Bulk and surface diffusion coefficients $\left(D_{v}\right.$ and $\left.D_{s}\right)$ plotted against $T_{g} / T$. The $D_{v}$ values nearly form a master curve, whereas $D_{s}$ values have stronger molecular dependence. $\mathrm{A}_{\mathrm{s}}$ upper bound from this work is shown for polyalcohols; Right: Correlation between the rates of surface crystal growth $u_{s}$ and surface diffusion (Adapted from Chen et al. 2016)

development. Molecules where the chemical space is restricted should be considered for continued ASD use; this would include drug substances with highly planar/ rigid molecules with high melting point $\left(>200{ }^{\circ} \mathrm{C}\right)$, very low solubility $(<0.1 \mathrm{mg} / \mathrm{mL})$, significant $\mathrm{pH}$ effects, low permeability, no prodrug handles, high propensity for supersaturation, and an inability to easily obtain crystalline forms. Many ASD properties need to be measured and understood to provide a smooth transition into development (including solubility, $\mathrm{T}_{\mathrm{g}}, \mathrm{T}_{\mathrm{m}}, \mathrm{T}_{\mathrm{c}}$, supersaturation potential, dissolution behavior, spray dry solvent solubility, ASD stability in aqueous suspension, and accelerated stability of the solid ASD). While many of these properties may be measured, the translation into larger scale production, formulation, and storage is not always routine.

Progressability and developability need to be considered and, for many systems, it will come down converting uncertainty into measurable risk. Measuring the necessary data to fully understand the risks for these systems continues to be a challenge and is an area where further work is needed. Using amorphous dispersions in novel areas may help to mitigate these risks and expand the use of amorphous materials. Future technologies

Increased Chemical Diversity Requires More Integrated Role of Drug Delivery During Lead Optimization

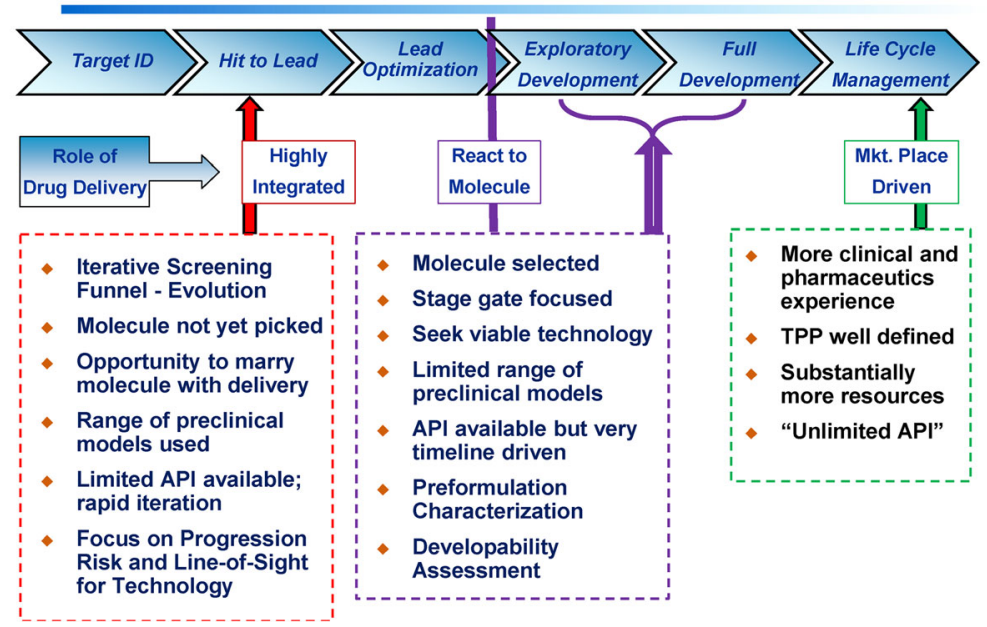

Fig. 3 Schematic of integrated role of drug delivery during lead optimization (Hageman, June LOL Conference, University of Wisconsin, June 2016) 
Table 1 Advantages and disadvantages of ASDs in drug discovery

\begin{tabular}{|c|c|}
\hline Advantages & Disadvantages \\
\hline $\begin{array}{l}\text { 1. ASD tends to normalize early drug substance discovery lots and in vivo } \\
\text { studies } \\
\text { a. Greater than } 50 \% \text { early discovery lots are amorphous } \\
\text { b. Residual solvent levels are actually lower with ASD than other solid } \\
\text { forms } \\
\text { c. More consistent formulatability for discovery dosing } \\
\text { d. More soluble form for use in combination with other solubilized } \\
\text { systems } \\
\text { e. Minimize pH and food effect variability preclinically (from single to } \\
\text { multidose) } \\
\text { f. Physical stability in dosing suspension is good surrogate for } \\
\text { crystallization propensity } \\
\text { 2. Provides relatively physiologically benign vehicle for drug delivery } \\
\text { a. Pre-GLP high dose toxicokinetic studies and multiday toxicology studies } \\
\text { b. GLP toxicology studies } \\
\text { 3. Provides a relatively benign vehicle for drug delivery in Gl disease } \\
\text { models } \\
\text { a. Infectious bowel disease (Crohn's disease, colitis) } \\
\text { b. Potential for minimally upsetting enteric flora? }\end{array}$ & $\begin{array}{l}\text { 1. Known Knowns } \\
\text { a. Preclinical to clinical to commercial product transition less well } \\
\text { understood } \\
\text { b. Lacking surrogate assays with reliable translation } \\
\text { 2. Known Unknowns } \\
\text { a. Selecting for a molecule which subsequently requires ASD for } \\
\text { delivery } \\
\text { i. Risk of failure due to crystallization on storage } \\
\text { ii. Limited room for dose escalation due to limited drug loading } \\
\text { b. Less ability to mitigate chemical liability } \\
\text { c. More resource intensive at both GLP and GMP stages than } \\
\text { conventional products } \\
\text { i. Longer timelines for progression? } \\
\text { ii. Greater cost of goods? } \\
\text { 3. Unknown Unknowns } \\
\text { a. Potential for unexpected ADME and toxicology properties } \\
\text { i. Unexpected distribution and clearance? } \\
\text { ii. In vivo crystallization? (locally/systemically) } \\
\text { iii. Other }\end{array}$ \\
\hline
\end{tabular}

include 3D printed dosage forms, combined technologies (such as spray layered ASD on microparticulates), delivery of prodrugs as ASDs, and parenteral applications using acceptable polymers and liposomal or phospholipids.

\section{Amorphous structure and characterization Amorphous content and structure}

As described above, ASDs have been extensively used for delivery of poorly soluble drugs to enhance dissolution and improve bioavailability. The first step to formulating ASDs is to have a thorough understanding of the amorphous solid forms of a drug substance.

Professor Kenneth Morris, Long Island University, gave a presentation on the influence of molecular anisotropy on the structure and structural relaxation of amorphous solids. Dr. Morris started by stating what we know and agree on based on our current understanding of the amorphous solids. He stated that the evolution of spatial heterogeneity in amorphous solids leads to crystallization, that some characteristic cooperatively rearranging regions (CRRs) make up the solid, and that with time the solid relaxes to a more energetically favorable arrangement of molecules. He then proposed to extend the concept of molecular packing patterns to non-crystalline amorphous solids. For example, microstructures can form due to ordering based on density (toward crystallization) and bonds (toward locally preferred structures).

There is, however, disagreement on the size or even the nature of the clusters and fraction of interstitial space in amorphous solids, the origin of enthalpy for relaxation, the contribution of molecular geometry to the structure of amorphous solids and its relationship to relaxation, and finally the structural connection between the relaxation process and crystallization. There are possible implications of the molecular shape and packing on the size and nature of the CRRs in amorphous solids. The enthalpy relaxation is dependent on inter-CRR densification with persistent intra-CRR molecular packing such that the maximum size of CRRs is set as a limit for "stable" amorphous solids.

Dr. Nara Variankaval, Merck, discussed the distinction between the amorphous phase and defects in the solid. For example, as crystals are milled or compacted progressively smaller, "crystalline domains" can be produced and defects accumulated due to shear and compression. In this manner, amorphization can be considered as the extreme limit of the defect density (Bates et al. 2006). There are two competing phenomena for this amorphization to occur. One is diffusion and the other is ballistic jump, which is a term used for disordering produced by external forces (Willart and Descamps, 2008). At temperatures lower than the $T_{g}$, the process of amorphization is preferred as longer times are required to anneal any defect/disorder or amorphous content into the crystal. The most common way of affirming the amorphous glassy state is by having a clear $\mathrm{T}_{\mathrm{g}}$ in the heating curve of the DSC and the absence of Bragg peaks in the X-ray diffraction pattern. Other techniques for detecting phase change are used and with their advantages and shortcomings as shown in Table 2. The best approach for detecting phase changes is to use combination of orthogonal techniques with different sensitivities.

In general, most of the processing in the pharmaceutical industry is conducted at room temperature or higher. These processes include milling and compaction where temperature rises are often observed. This can result in higher chances of annealing. Defects and amorphous contents can have similar consequences. In general, it is more important to determine the "extent" of disordering than the type of disordering. There are many aspects of the disordering that need to be 
Table 2 Methods used to detect and differentiate amorphous state and defects

\begin{tabular}{|c|c|c|}
\hline Method & Advantages & Disadvantages \\
\hline $\begin{array}{l}\text { XRPD and Rietveld } \\
\text { Modeling }\end{array}$ & $\begin{array}{l}\text { - Reitveld analysis can be used to understand peak } \\
\text { broadening due to size/strain or amorphous content }\end{array}$ & $\begin{array}{l}\text { - Requires careful deconvolution of size and strain effects and the } \\
\text { assessment of instrumental broadening }\end{array}$ \\
\hline DSC & $\begin{array}{l}\text { - Simple } \\
\text { - Change in heating ramps to delineate differences between } \\
\text { in "true" amorphous state and defects }\end{array}$ & $\begin{array}{l}\text { - Variable response from different PSD fractions due to small size } \\
\text { - Recrystallization/annealing depends on the condition of the run } \\
\text { and extent of cooling }\end{array}$ \\
\hline DEA & $\begin{array}{l}\text { - Can show typical glassy state signatures - peak loss and } \\
\text { beta relaxation } \\
\text { - Response vs. Hz can help delineate the type of transition }\end{array}$ & $\begin{array}{l}\text { - Overlapping signals may be difficult to deconvolute if multiple } \\
\text { transitions occur in the same temperature range }\end{array}$ \\
\hline SSNMR & $\begin{array}{l}\text { - Molecular level picture } \\
\text { - Relaxation time maps can help differentiation }\end{array}$ & $\begin{array}{l}\text { - Long acquisition times in only }{ }^{13} \mathrm{C} \text { are available } \\
\text { - Labeling may be required to deconvolute origins of transitions } \\
\text { at the molecular level } \\
\text { - Indirect indication of structure through relaxation time } \\
\text { measurements }\end{array}$ \\
\hline $\begin{array}{l}\text { Pair Distribution } \\
\text { Function analysis }\end{array}$ & $\begin{array}{l}\text { - Molecular level picture of the phase } \\
\text { - More sensitive to local structure/order }\end{array}$ & $\begin{array}{l}\text { - Careful analysis of data is required } \\
\text { - Molecular models may be needed for getting a complete } \\
\text { picture }\end{array}$ \\
\hline
\end{tabular}

considered. For example, it is possible that defects and disorders could have as deleterious an impact as amorphous content. This is due to the presence of activated surfaces in defects with higher energy and therefore higher potential for interactions with excipients in drug products.

\section{New tools for amorphous form characterization}

Dr. Evgenyi Shalaev, Allergan, presented a panorama of new characterization tools for understanding amorphous structure and properties. The standard characterization methods for solid state properties include XRPD (crystallinity), DSC (mobility, crystallinity), and water/solvent content, and are amongst many that are used across the pharmaceutical industry (Guo et al. 2013). The limitation of conventional XRPD for amorphous/crystalline physical mixtures is that it has only an approximately $1 \%$ detection limit for crystalline material. These standard techniques do not have the high resolution required to measure molecular mobility and structure to predict stability and rank-order.

Dr. Shalaev discussed several techniques with high sensitivity for identification of physical forms of drug substances and excipients at lower concentrations including:

- Small-angle X-ray scattering (SAXS) technique is applied in the pharmaceutical field to assess physical and chemical stability of samples by distinguishing between crystalline mesophase, amorphous, and crystalline forms. SAXS has also been used for optimization and troubleshooting of manufacturing processes such as freeze-drying.

- Synchrotron X-ray diffraction technique allows for phase analysis of complex mixtures, by resolving overlapping peaks drug substance and excipient mixtures. More recently, in techniques such as 2-D synchrotron X-ray diffraction, amorphous sucrose samples spiked with crystalline sucrose were shown to have crystalline diffraction peaks at $\sim 0.5 \%$ crystal concentration and lower.

- Neutron scattering studies of amorphous materials were shown to have similar applications to small/ wide angle $\mathrm{X}$-ray scattering techniques. In this technique, the differential scatter of deuterium (D) and hydrogen $(\mathrm{H})$ and their varying $\mathrm{D} / \mathrm{H}$ ratios allow extraction of detailed structural information without any chemical destruction by the $\mathrm{x}$-ray radiation.

Dr. Sheri Shamblin, Pfizer, gave a presentation focused on how are we are winning the war against thermodynamics of amorphous conversion of solid forms to the more stable crystalline forms. The specific question was how to overcome crystallization and chemical reactivity in amorphous pharmaceutical systems. The unwanted consequences of high energy and enhanced molecular motion in amorphous systems are due to coupling of physical and chemical change to molecular mobility, the complex nature of structure and dynamics, and crystallization tendency (Baird et al. 2010, Van Eerdenbrugh 2010). Molecular mobility and molecular volume increase from crystalline to amorphous to liquid forms. Therefore, there is potential for greater chemical reactivity in the amorphous state (Shamblin et al. 2006). Physical stability, however, is governed by thermodynamics and molecular mobility. As molecular mobility increases, the thermodynamic driving force decreases, and the increase in molecular mobility leads to crystallization. The type or mode of molecular mobility required for physical or chemical change needs to be determined.

The fragile nature of small molecule drugs leads to complex changes in structure and dynamics over 
relatively narrow ranges of temperature. Crystallization can deviate from measures of mobility in certain temperature ranges. In one example, Dr. Shamblin described crystallization tendencies of 50 drugs via experimental approaches that measure crystallization of amorphous forms and from supersaturated solutions using the solvent shift technique. A third of the compounds evaluated failed to crystallize after $1 \mathrm{~h}$, while the remaining compounds crystallized at intermediate time points. The crystallization kinetics of a compound can then be altered through the addition of excipients such as polymers or other substances that may act as inhibitors.

\section{Solubility enhancement with ASDs}

Professor, Lynne Taylor, Purdue University, talked about solubility enhancement using amorphous formulations. Dr. Taylor first focused on the concepts of solubility, supersaturation, and the connection to membrane transport rate. The basic mechanism of ASDs that rapidly dissolve to yield supersaturated solutions is not well understood. In theory, the solubility of an amorphous solid cannot exceed that of the crystalline material which is the thermodynamically stable form. However, if the crystallization is slow, then concentrations greater than the crystalline solubility can be achieved and, under some conditions, "a metastable equilibrium can exist between the amorphous material and the aqueous solution which is termed liquid-liquid equilibrium (LLE)" (Taylor and Zhang 2016). If the crystalline solubility is known, then the amorphous solubility can be estimated.

It is well known that the solubility of amorphous material is a kinetic phenomenon and is time dependent. Amorphous material dissolves over time to reach the equilibrium solubility of the thermodynamically stable crystalline form. Thus, the experimental determination of the amorphous solubility is very challenging. However, the most direct approach is very similar to that for the determination of the solubility of the crystalline form as the amorphous solid is added to aqueous medium and the concentration increase with time is monitored until a plateau is reached. For some compounds, good agreement has been observed between the experimentally observed amorphous solubility and the predicted value. However, many systems crystallize prior to attainment of the amorphous solubility, leading to large discrepancies between predicted and experimentally observed values. This has led to the development of alternative approaches. The amorphous solubility advantage for 23 compounds is shown in Fig. 4 which shows the predicted versus experimental amorphous-to-crystalline solubility $(\mathrm{a} / \mathrm{c})$ ratios using in situ generation of amorphous material (Taylor and Zhang 2016).

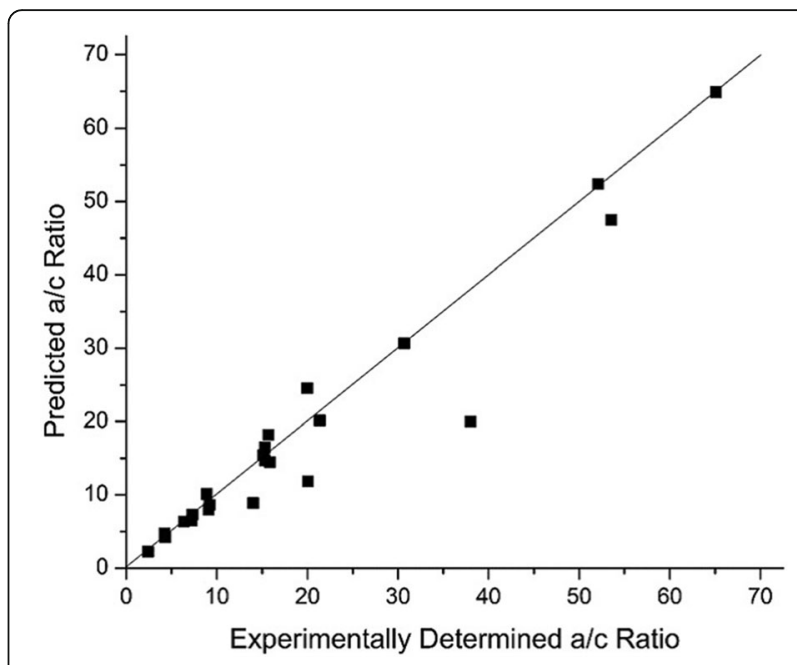

Fig. 4 Predicted versus experimental amorphous-to-crystalline solubility (a/c) ratios (Adapted from (Taylor and Zhang 2016))

Molecular dynamics simulations and screening for ASDs Dr. Yi Gao, Abbvie, presented research on molecular dynamics (MD) simulation which takes its roots from quantum mechanics. On study showed that drug-polymer interactions in the context of crystallization inhibition using MD simulations is a powerful tool to discern molecular level interactions in order to determine the energetics involved in the drug-polymer interactions in aqueous media. The crystal growth of tolazamide (TLZ) is modulated by the presence of a diblock copolymer, poly(ethylene glycol)block-poly(lactic acid) (PEG-b-PLA). The crystal morphology of the drug then changes from needles to plates in aqueous media. Molecular dynamics simulations on crystal surfaces of TLZ in water containing PEG-b-PLA were conducted to better understand the crystal surface drug-polymer interaction. Interaction of PEG-b-PLA with the (001) face occurred more rapidly ( $\leq 10 \mathrm{~ns}$ ) and more strongly than that with the (010) face, and there was little interaction with the (100) face. Hydrophobic and van der Waals (VDW) interactions were the dominant forces, accounting for more than $90 \%$ of total interaction energies. The work suggests that polymers capable of forming strong hydrophobic and vdW interactions might be more effective in inhibiting crystallization of poorly water-soluble and hydrophobic drugs in aqueous media (such as gastrointestinal fluid) than those with hydrogen-bonding capacities. Such in-depth analyses and understanding facilitate the rational selection of polymers in designing supersaturation-based enabling formulations. These types of simulations are essential for understanding molecular interactions of ASDs.

\section{Manufacturing ASDs and case studies} Particle engineering of amorphous solid dispersion Dr. Dan Miller, Novartis, shared examples of how understanding and controlling molecular mobility in 
amorphous materials can result in the development of room temperature stable powders for pulmonary drug delivery. Based on the research efforts of Professor Zografi and his colleagues, Dr. Miller demonstrated how to construct a "stability map" to help understand the relationships between environmental variables, such as $\mathrm{RH}$ and temperature, and the relaxation timescales in the region at and around the glass transition of the product produced by spray drying. Once the stability map is known, packaging can be designed to maintain the stability over the shelf life of the product as well as in-use stability in the hands of the patient. Dr. Miller noted that the characteristic relaxation time of $300 \mathrm{~h}$ is commensurate with the "Tg-50 K" rule of thumb for long term stability. This "rule of thumb" was introduced by Professor Zografi in the 1990 s and is thought to represent the point at which molecular mobility is sufficiently slowed due to increased viscosity such that crystallization is inhibited. Therefore, as many formulators working with amorphous solid dispersions know, a prudent storage temperature for an ASD is $\mathrm{Tg}-50 \mathrm{~K}$.

The selection of excipients combined with optimized spray drying process conditions can result in low density engineered particles with either a stable amorphous center surrounded by a precipitated shell or a whiffle ball geometry. The surface enrichment/composition for each type of engineered powder can be described by use of the Peclet number. Pulmonary dry powders containing insulin $\left(\right.$ Exuber $\left.^{\circ}\right)$ were prepared from a spray solution and were shown to be stable for years if the RH (and thus water content) and temperature were controlled. Physical stability was modeled by characterizing the $T_{g}$ as a function of water content using the Gordon Taylor equation and molecular mobility as monitored by relaxation time. The chemical stability of insulin was monitored by stretched kinetics. The time, temperature, and
$\mathrm{RH}$ conditions that the powder and product were exposed to during the production process, testing and evaluation, and patient handling were added to the overall solid-state phase behavior diagram for tobramycin spray dried powders. Viscosity changes relative to the $T_{g}$ were characterized by using the Williams, Landel, Ferry (WLF) equation to understand the conditions at which viscous flow of amorphous tobramycin would occur to explain the degradation in aerosol performance (Fig. 5).

Dr. Miller demonstrated the practical utility of relationships that drive the chemical and physical stability in the amorphous state. Through our understanding of the impact of water, temperature, and time on the solid state, engineered amorphous powders designed for use in pulmonary drug products can be developed and marketed. This level of understanding was not possible before Professor Zografi initiated his research in this field and therefore his efforts (as well as those of his colleagues) have benefited not only the researchers, but patients.

\section{Process-induced disorder and transformations in drug product manufacturing}

Dr. Sarah Nicholson, Bristol-Myers Squibb, discussed the advantages and disadvantages of developing products with amorphous content. Drug product manufacturing processes can either generate amorphous material intentionally or unintentionally. Process controls can be employed to avoid unintended transformations and two case studies were presented to demonstrate this point.

Intentional production of amorphous transformations is generally the goal when manufacturing processes using hot melt extrusion (HME) or spray drying. Confocal Raman microspectroscopy is a technique that can be used to assess homogeneity in the solid state. As an example, Dr. Nicholson discussed a HME dispersion that

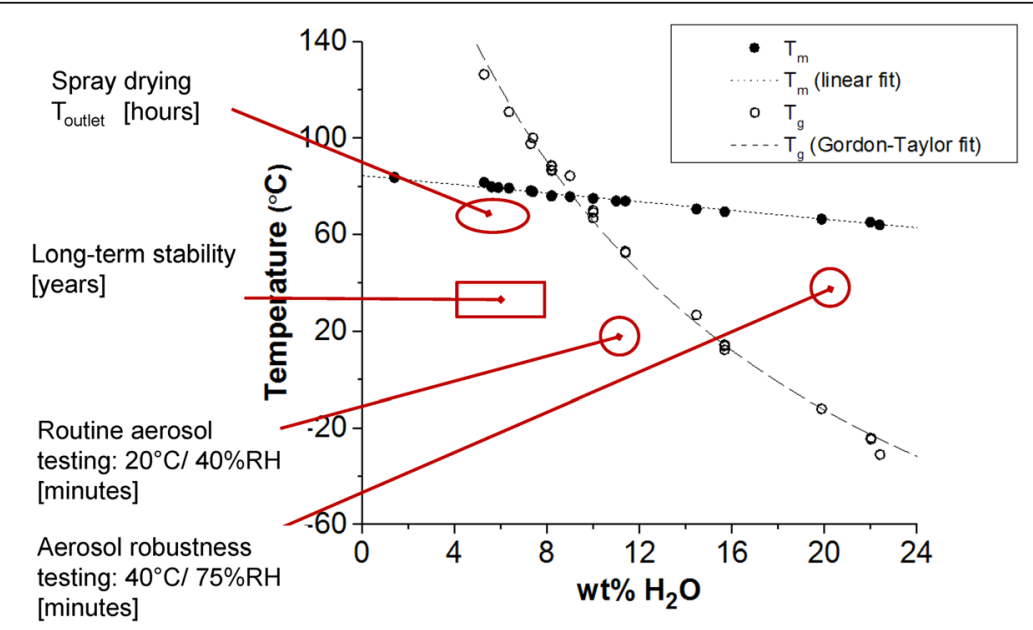

Fig. 5 Tobramycin Inhalation Powder (TIP) state diagram: Impact of water on the solid state (Adapted from Miller et al. 2017) 
was shown to be amorphous by DSC and XRPD. However, a scan using confocal Raman microspectroscopy showed that the dispersion prepared at low screw speed was heterogeneous, thus leading to a higher probability to crystallize on storage. The same dispersion, when processed using a fast screw speed, was shown to be homogeneous. The heterogeneous dispersion was shown to crystallize whereas the homogeneous dispersion remained amorphous after 3 months of stability storage.

The benefits of Raman spectroscopy as an inline technique to monitor crystalline form and content from tablets prepared from a wet granulation process and a spray dried dispersion (SDD) process was discussed. A wet granulation case study demonstrated the impact of water content, temperature, and wet massing time on the solid form of a highly soluble drug substance. Near IR and Raman probes were used as inline tools to assess the water content and solid form during granulation. Interestingly, the high solubility of the drug substance resulted in the production of unintentional amorphous content as well as an increase in hydrate formation due to the water addition. The kinetics of the transformation were monitored using Raman and the conversion increased with increasing temperature and water content (Fig. 6). The rate of drying also impacted the final solid form of the drug substance, with fast (fluid bed) drying resulting in mostly amorphous material and slow (tray) drying causing a reduction in the amorphous content. It was concluded that an in-line tool such as Raman spectroscopy is a simple and sensitive technique able to assist in both formulation optimization and process development. As discussed throughout the conference, molecular mobility is accelerated with temperature and moisture content. Increasing water content for amorphous solids decreases the glass transition temperature and thus increases the propensity for crystallization.

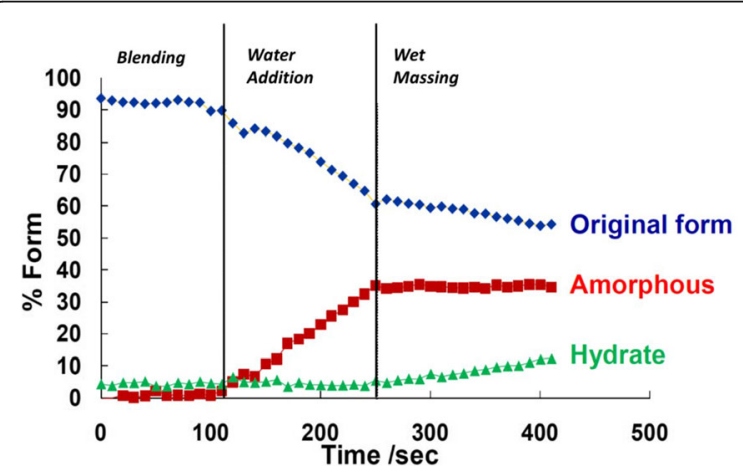

Fig. 6 Monitoring the kinetics of transformation during processing using Raman spectroscopy (adapted from S. Nicholson, June LOL Conference, University of Wisconsin, June 2016)
Performance tests in early formulation development of amorphous dispersions

Michael Grass, Capsugel, presented a summary of early formulation performance screening techniques and followed this with three associated case studies. The screening techniques included: speciation, amorphous solubility, Maximum Absorbable Dose (MAD), dissolution, and member flux. These performance tests provide input regarding the likelihood of success and allow for a rank ordering of formulations. An amorphous formulation development map (plots of $\mathrm{T}_{\mathrm{m}} / \mathrm{T}_{\mathrm{g}}$ vs. $\log \mathrm{P}$ ) can be used as a guide to identify the key formulation challenges associated with a specific compound. Drugs with high $\mathrm{T}_{\mathrm{m}} / \mathrm{T}_{\mathrm{g}}$ $(\mathrm{K} / \mathrm{K})$ values tend to be fast crystallizers, while those with lower ratios tend to be slow dissolvers. Therefore, fast crystallizers will require polymers that provide a "parachute" while the slow dissolvers require a "spring". Formulation of ASDs typically involves the addition of polymers to raise the $T_{g}$, enhance the dissolution, and protect the drug from crystallizing. Polymer selection can be optimized by characterizing the interactions between the polymer and drug and measuring the amorphous solubility in various polymer systems. The approach to measuring amorphous solubility is described in Figs. 7 and 8. Quantifying the amorphous solubility allows for an understanding of the solubility enhancement provided by an amorphous solid dispersion.

Various formulations can be ranked by evaluating them in a microcentrifuge dissolution test to characterize the dissolution of various species in the dissolution milieu (Fig. 9), and a boundary layer limited membrane test aimed at measuring the permeability of the various species. From these data, the MAD of the amorphous and crystalline forms can be estimated. The magnitude of the MAD relative to the clinical dose provides the formulator with and understanding the potential success of various processing options, such as particle size reduction and spray drying.

Three case studies were presented. Itraconazole, with a $\mathrm{T}_{\mathrm{m}} / \mathrm{T}_{\mathrm{g}}$ of 1.35 and a $\log \mathrm{P}$ of 5.9 was determined to have an amorphous solubility 100 times the crystalline form of the drug. Utilizing the various formulation screening tools, it was determined that a colloidal dispersion of the drug would be the most promising formulation approach. Ketoconazole, with a $\mathrm{T}_{\mathrm{m}} / \mathrm{T}_{\mathrm{g}}$ of 1.34 and a $\log \mathrm{P}$ of 4.3 was determined to have an amorphous solubility $\sim 20$ times that of the crystalline form of the drug. The polymer hydroxypropylmethylcellulose acetate succinate (HPMCAS-M) was shown to inhibit crystallization and sustain the amorphous solubility in FaSSIF with a predominant species being drug in micelles. Erlotinib, with a $T_{m} / T_{g}$ of 1.42 and a $\log \mathrm{P}$ of 3.0, was determined to have an amorphous solubility $\sim 20$ times that of the crystalline drug. Erlotinib is known to rapidly precipitate on transfer from gastric to 


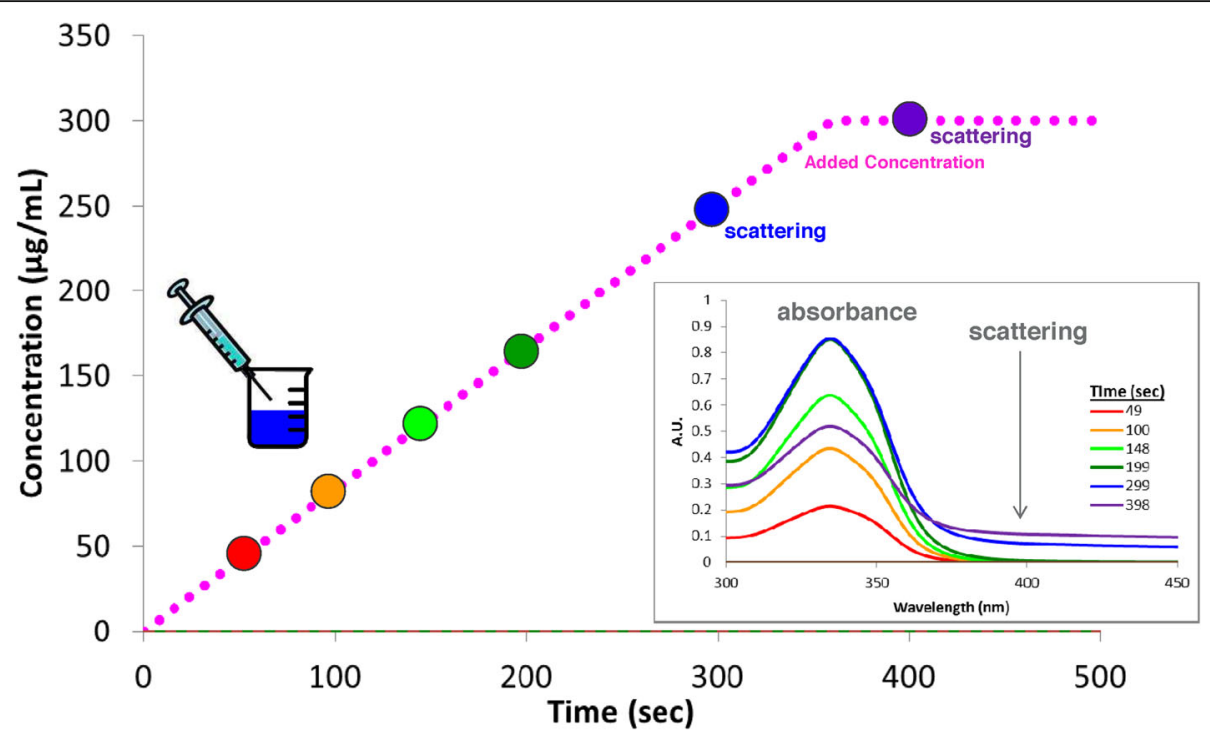

Fig. 7 Amorphous solubility determination (reproduced with permission from Capsugel)

intestinal media. Precipitation was delayed in FaSSIF when the drug was prepared in an HPMC capsule. Solubility studies in the presence of HPMC confirmed a "parachute" effect.

\section{Related areas: Mesoporous supports, Mesophases, and Cocrystals}

Another method of stabilizing amorphous drug substances involves the use of mesoporous supports, as discussed by Professor Robin Bogner, University of Connecticut. Mesoporous supports include amorphous silicates with pore diameters between 2 and $50 \mathrm{~nm}$ (such as Neusilin US2, Sorbent 62,700, and Silysia 470 and 350). The scaffold pore structure thermodynamically stabilizes the amorphous drug at supersaturated concentrations (Qian and Bogner 2011, Qian et al. 2011). Smaller pore sizes $(<15 \mathrm{~nm})$ show a higher capacity for amorphization. Drugs can be deposited by vapor, melt, or solution methods, and studies report deposition of naphthalene, ibuprofen, quinapril, TAS-301, and itraconazole. The mesoporous scaffolds come with limitations, such as storage at low relative humidity $(\mathrm{RH})$ (water will replace drug rendering them unstable) and chemical reactions with acid-labile and oxidationlabile drugs. However, bioavailability can be enhanced, possibly due to the supersaturated solution produced by the amorphous material, water replacing the drug in the mesoporous support resulting in rapid dissolution, or the silicate improving wetting properties below the amorphization capacity.

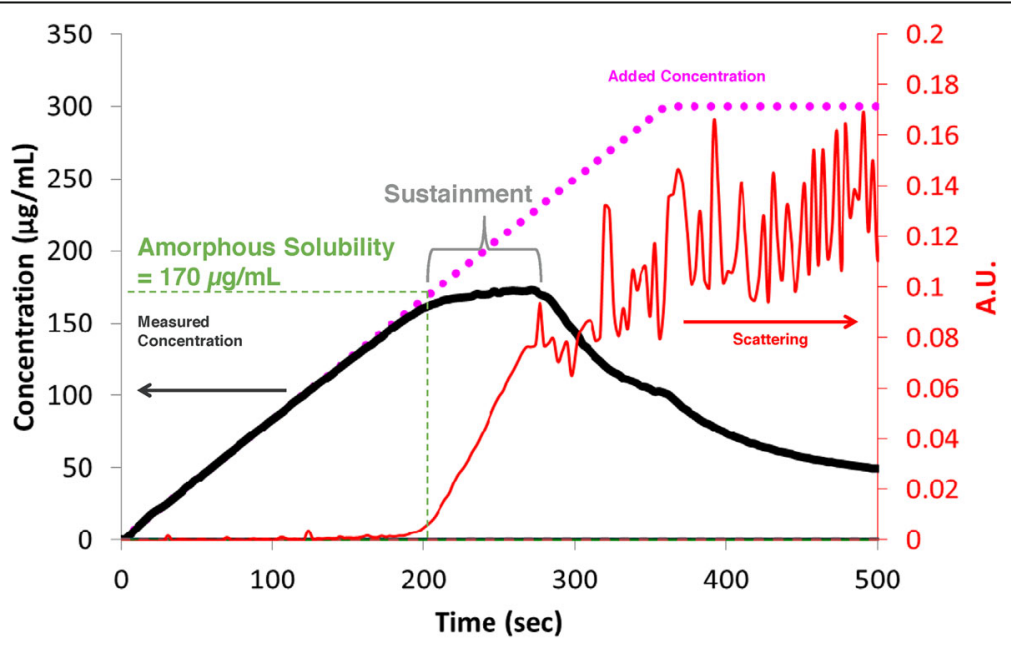

Fig. 8 Determining the impact of formulation on the "parachute" effect (reproduced with permission from Capsugel) 


\section{Contribution to Absorption}
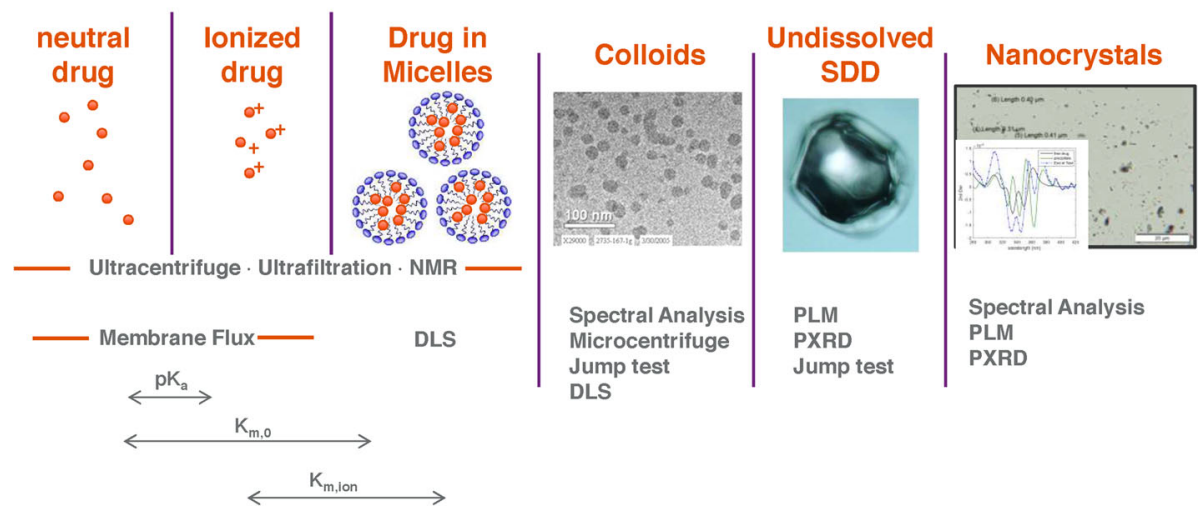

Spectral Analysis

PLM PXRD

Fig. 9 Speciation of dissolved and suspended drug species (reproduced with permission from Capsugel)

Dr. David Lechuga-Ballesteros, Pearl Therapeutics, discussed mesophases, which are the phases between amorphous and crystalline materials. Liquid crystals, one example of a mesophase, exhibit order in two dimensions, but not in three dimensions as observed with crystalline materials. Different liquid crystalline phases are known, and nematic and smectic phases are commonly observed for pharmaceutical systems. Several drugs exhibit liquid crystalline phases including cromolyn (nematic and smectic), cyclosporine (smectic), folic acid (smectic), fenoprofen (smectic), itraconazole (nematic), leuporolide (nematic), methotrexate (nematic), and tobramycin (nematic). Solid state characterization methods can be used to investigate the possible occurrence of liquid crystalline states. While an amorphous halo and $\mathrm{T}_{\mathrm{g}}$ would indicate an amorphous material, the presences of birefringence and diffraction peaks in small angle X-ray scattering (SAXS) would confirm the liquid crystalline nature of a solid (Wunderlich 1999). These materials can display various phase transitions, as shown in Fig. 10. The existence of mesophases should be confirmed to explain "unusual" behavior of amorphous solids.

A third technology to increase solubility and change drug substance properties are cocrystals, which are crystalline materials containing a drug substance and a coformer (usually counterions used for salt formation) where both components are neutral and there is no
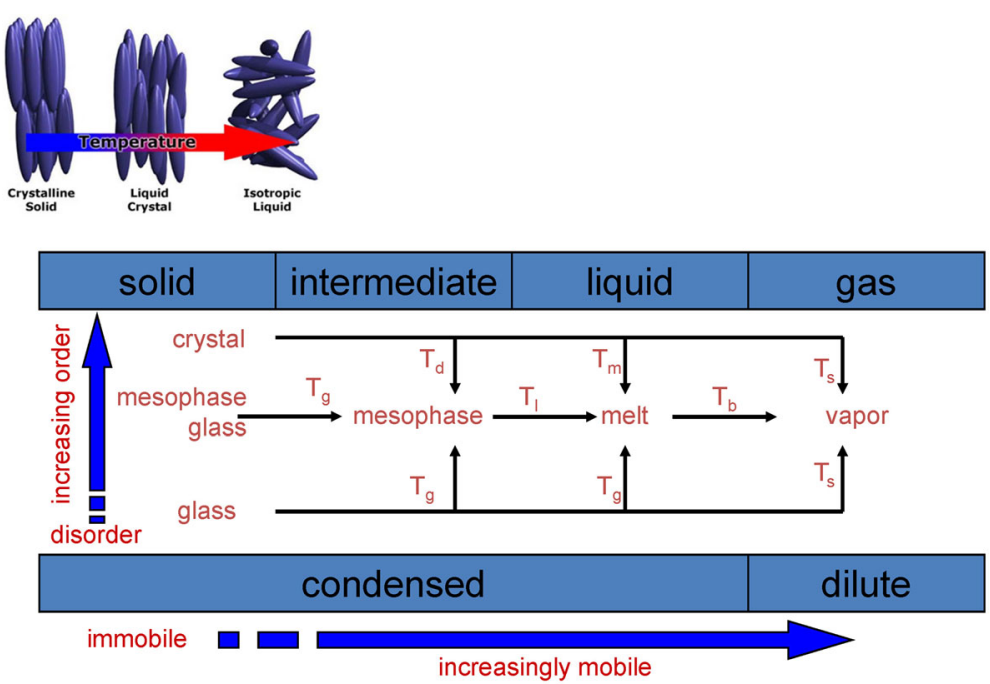

Fig. 10 Schematics of the transitions between ordered and disordered solid systems in a one-component system (adapted from Wunderlich 1999) 
proton transfer. A draft FDA guidance in 2011 resulted in significant discussion within the pharmaceutical community. The guidance stated that polymorphs of a drug substance were considered the same active ingredient for a regulatory filing, and salts were considered a different active ingredient, while cocrystals were classified a drug product intermediate. A second provision in the guidance stated that NDA/ANDA applicants needed to provide supporting data for a cocrystal designation covering proof of cocrystal formation and assurance of cocrystal dissociation prior to reaching the site of action. During the comment period, many negative responses were received covering numerous areas of development. IQ convened a consortium of industry scientists to engage the FDA to better understand the Agency's perspective on the guidance and provide a unified industry perspective on how to develop cocrystals. The IQ position paper covered several development areas, such as characterization, manufacturing sites (drug substance vs. drug product), supply chain management, stability studies, expiry dating, and regulatory filings. It was recommended that cocrystals be classified in the same category as solvates and polymorphs of a drug substance (similar to the European Medicines Agency (EMA) position), thus classifying them as the same active ingredient. As a post meeting note, the FDA did revise the guidance in August 2016 and cocrystals are now considered the same active ingredient, the same designation as polymorphs and solvates (FDA 2016).

\section{The BCS and amorphous solid dispersions} The application of BCS based biowaiver in drug regulatory review

Dr. John Duan, FDA, presented a review of the BCS system (Fig. 11) and the utility of the biowaiver system based on the classification system. He emphasized that the qualitative and quantitative risk based approach used by the FDA when assessing biowaiver applications and provided a window into the common deficiencies encountered. Dr. Duan also provided an overview of the 2015 changes proposed in the BCS Guidance document (FDA 2015).

The co-chairs for the CDER BCS committee are Lawrence $\mathrm{Yu}$ and Mehul Mehta with membership from OPQ/ONDP/Division of Biopharmaceutics, OTS/Office of Clinical Pharmacology, and OGD/Office of Bioequivalence. When a sponsor submits a biowaiver request a primary reviewer is assigned and that reviewer distributes the package to the committee. The committee determines whether to grant the biowaiver based on a majority vote and that decision is conveyed to the sponsor. Since 2003, a total of 68 cases have been reviewed, $69 \%$ of which were determined to be BCS 1 drugs.

Some common deficiencies in the content of biowaiver applications included:

- Insufficient number of $\mathrm{pH}$ conditions for solubility profile

- Incomplete information on solubility data

\section{BCS Classes}

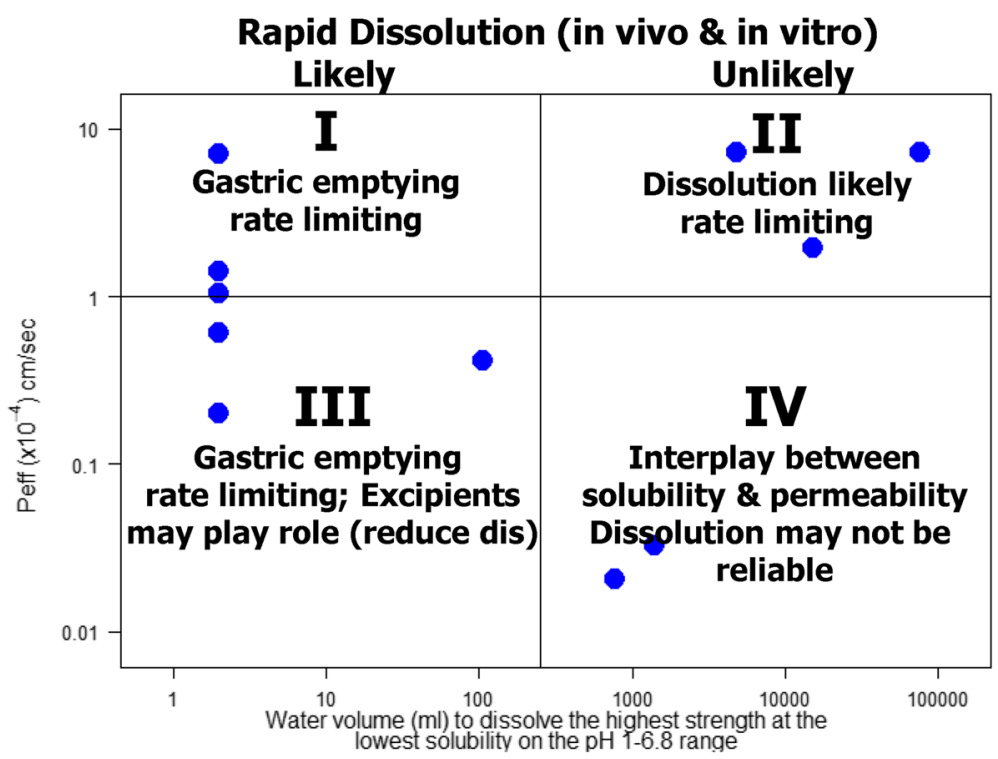

Fig. 11 BCS classes and dissolution behavior (Hussain 2000) 
- Lack of information on the stability indicating assay for analyzing solubility samples

- Missing $\mathrm{pK}_{\mathrm{a}}$ information

- Inadequate validation of the permeability test method

- Incomplete details or lack of data on the gastrointestinal stability of drug,

- Lack of gastrointestinal stability data when less than $85 \%$ of the drug is excreted unchanged in urine

- Lack of dissolution information (e.g., missing information on dissolution media volume, individual dissolution data, multimedia dissolution, etc.)

Dr. Duan described the changes proposed in the 2015 BCS Guidance draft. These changes included:

- An extension of biowaivers to BCS Class 3 drugs also (with Q1, Q2 restrictions)

- Addition of "very rapid" dissolution criteria ( $>85 \%$ in 15 min)

- A change in the permeability boundary from 90 to $85 \%$

- A change the $\mathrm{pH}$ solubility range from 1 to 7.5 to $1-6.8$.

- Paddle speed changed

- Volume of dissolution media changed

- Additional topics/clarification on FDCs (Fixed Dose Combinations), ODTs (Orally Disintegrating Tablets), products

- Update to the list of model drugs

- Strengthen GI stability requirement

The value of the BCS is not only awarding biowaivers. Knowing the BCS class also helps the pharmaceutical scientist select excipients, define formulation composition, and select manufacturing approaches that address the physicochemical limitations of the drug substance. Understanding that the compound being developed is dissolution limited due to dose and/or solubility typically requires conversion of the crystalline solid to that of a high energy amorphous solid or a cocrystal. Therefore, the BCS is not just based on sound scientific and regulatory principles, but it is a valuable tool for drug development.

\section{Product development using BCS principles and a parachute}

Dr. Jayne Hastedt, JDP Pharma Consulting, LLC presented "Product Development Using BCS Principles and a Parachute" and included a question and a controversial hypothesis in her discussion. The question raised was: "Can processing of a BCS 2 drug substance using particle engineering/formulation approaches that result in an increased rate of dissolution and an enhanced "apparent" solubility of the product impact the classification of a drug substance within the BCS system?" The associated hypothesis discussed was: "Formulation and processing approaches used for BCS 2 drugs that result in drug products with characteristics similar to BCS 1 drugs should allow for biowaivers for these low solubility drug substances." If this hypothesis could be supported, expensive in vivo clinical studies linking formulations and be eliminated. By formulating the drug in a stable amorphous state, "sameness" of two formulations from various stages of development can be determined by using in vitro dissolution testing. This approach would streamline product development and even generic product approval allowing patients faster access to medications. Currently there is no precedent for a BCS 2 drug substance to be granted a biowaiver. BCS 2 drugs have low equilibrium solubility values between $\mathrm{pH} 1$ and 6.8 and a high dose number and low dissolution number. Biowaivers are typically provided for BCS 1 and, under the latest draft guidance (FDA 2015), for BCS 3 compounds.

Formulation development and processing of a low solubility/high permeability BCS 2 compound to produce an amorphous solid dispersion alters the physicochemical properties of the drug to the extent that it behaves like a BCS 1 compound. If the resulting oral dosage form can maintain a high degree of supersaturation in vivo during the absorption window in the body ( $4 \mathrm{~h}$ by BCS standards), then in vivo behavior should be similar to a BCS 1 drug. This is described as a "spring and parachute" approach as shown in Fig. 12 (Brouwers et al. 2009). There are multiple in vitro and in vivo examples in the literature demonstrating both the dissolution rate and bioavailability improvements associated with BCS 2 drugs when formulated as amorphous solid dispersions and even co-crystals (Bevernage et al. 2011; Friesen et al. 2008; Law et al. 2004; Engers et al. 2010; Bak et al. 2008). The in vitro dissolution of compound 1 shows a classic "spring and parachute", when formulated with HPMCAS-M as an amorphous solid dispersion. When evaluated in vivo in humans, the overall enhancement in bioavailability for the various compounds evaluated ranged from 2- to 40 -fold relative to the crystalline form of the drug (Fig. 13).

After this presentation, a panel discussion was held to address the comments and questions from the audience. Although in vitro and in vivo data exist for co-crystals and amorphous solid dispersions demonstrating an increase in apparent solubility and bioavailabilty, it was noted that the intrinsic solubility of the drug is not altered. Since biowaivers are granted based on a riskbased scientific evaluation, a risk mitigation approach to provide further evidence to demonstrate the engineered drug's behavior in vivo would be useful. 


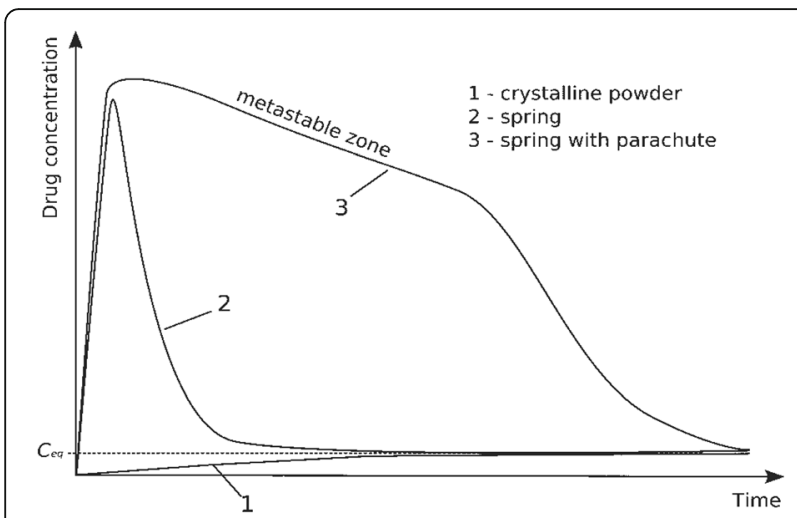

Fig. 12 Polymers can be used to generate a "spring and parachute" for amorphous solid dispersions (Adapted from Brouwers et al. 2009)

\section{Conclusions}

Professor Zografi's research laid the foundation for the current studies routinely performed for amorphous materials and ASDs in drug development pipelines, as well as the novel research being performed in these areas. Based on his groundbreaking work, it has become common place in the pharmaceutical industry to characterize, understand, and develop these materials.
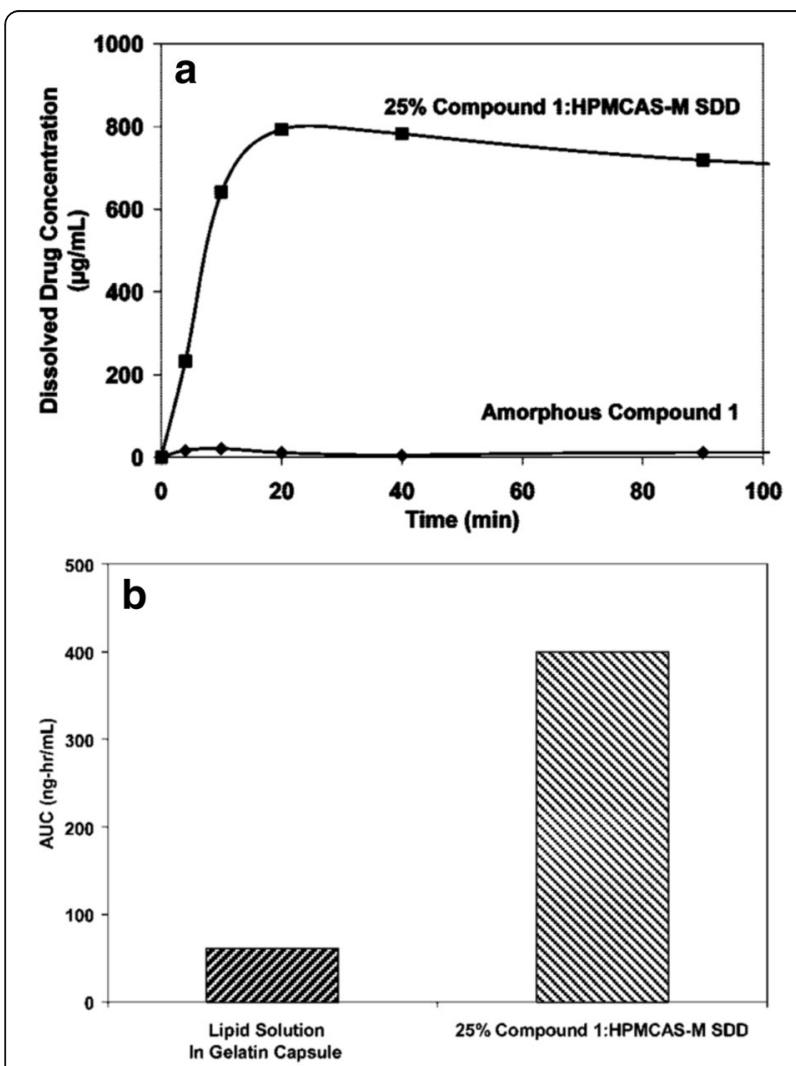

Fig. 13 The "spring and parachute" in vitro dissolution profile (top panel a) enables in vivo bioavailability enhancement (bottom panel b) (Adapted from Friesen et al. 2008)
The number of marketed products containing ASDs continues to grow and is an example of successfully transforming academic ideas into marketed products. Current academic research will continue to increase our understanding of these materials and will be used to more efficiently develop these systems into marketable products in the future.

\section{Abbreviations}

ADME: Adsorption, distribution, metabolism, and excretion; ANDA: Abbreviated New Drug Application; API: Active pharmaceutical ingredient; ASD: Amorphous solid dispersion; B.S.: Bachelors of Science; BCS: Biopharmaceutical Classification System; CDER: Center for Drug Evaluation and Research; CRR: Cooperatively rearranging regions; D: Deuterium; DEA: Dielectric analysis; $D_{s}$ : Surface diffusion; DSC: Differential scanning calorimetry; $D_{v}$ : Bulk diffusion; EMA: European Medicines Agency; FaSSIF: Fasted state simulated intestinal fluid; FDA: Food and Drug Administration; Gl: Gastrointestinal; GLP: Good Laboratory Practices; GMP: Good Manufacturing Practices; H: Hydrogen; hGh: Human growth hormone; HME: Hot melt extrusion; HPMC: Hydroxypropylmethylcellulose; HPMCAS: Hydroxypropylmethylcellulose acetate succinate; IQ: International Consortium for Innovation and Quality in Pharmaceutical Development; LOL: Land O'Lakes; M.S.: Masters of Science; MAD: Maximum absorbable dose; MD: Molecular dynamics; mg: Milligram; mL: Milliliter; NDA: New Drug Application; OGD: Office of Generic Drugs; OPQ/ONDP: Office of Product Quality/Office of New Drug Products; OTS: Office of Translational Sciences; PEG-b-PLA: Poly(ethylene glycol)-block-poly(lactic acid; Ph.D.: Doctorate of Philosophy; PLM: Polarized light microscopy; PSD: Particle size distribution; PVP: Polyvinylpyrrolidone; RH: Relative humidity; SAXS: Small angle X-ray scattering; SDD: Spray dried dispersion; SSNMR: Solid-state nuclear magnetic resonance; $T_{c}$ : Crystallization temperature; $T_{g}$ : Glass transition temperature; TIP: Tobramycin inhalation powder; TLZ: Tolazamide; $T_{m}$ : Melting temperature; $u_{s}$ : Rate of surface crystal growth; $v d W$ : van der Waals; WLF: Williams, Landel, Ferry; XRPD: X-ray powder diffraction

\section{Acknowledgements}

Two figures were reproduced from presentations at the 2016 Land of Lakes Conference with the permission of the respective authors: Fig. 3 (Hageman) Fig. 6 (Nicholson).

\section{Authors' information}

Ann Newman.

AN is currently a pharmaceutical consultant at Seventh Street Development Group and the Vice President of Scientific Development at Crystal

Pharmatech.

Jayne E Hastedt.

JEH is the Managing Director and Co-Founder of JDP Pharma Consulting, LLC, which provides CMC consulting services to the pharmaceutical and biotech industries with an emphasis on drug delivery. Mehran Yazdanian.

Mehran Yazdanian is the Sr. Director of Pharmaceutics at Teva Branded Pharmaceutical Products R\&D Inc.

\section{Authors' contributions}

All authors contributed equally to the writing of this meeting report. All authors read and approved the final manuscript.

\section{Competing interests}

The authors declare that they have no competing interests.

\section{Publisher's Note}

Springer Nature remains neutral with regard to jurisdictional claims in published maps and institutional affiliations.

\section{Author details}

${ }^{1}$ Seventh Street Development Group LLC, Kure Beach, NC 28449, USA. ${ }^{2}$ JDP Pharma Consulting, LLC, San Carlos, CA 94070, USA. ${ }^{3}$ Pharmaceutics Department, Teva Branded Pharmaceuticals R\&D, West Chester, PA 19380, USA. 
Received: 12 May 2017 Accepted: 26 July 2017

Published online: 11 August 2017

\section{References}

Ayenew Z, Paudel A, Rombaut P, Van den Mooter G (2012) Effect of compression on non-isothermal crystallization behavior of amorphous indomethacin. Pharm Res 29:2489-2498

Baird JA, van Eerdenbrugh B, Taylor LS (2010) A classification system to assess the crystallization tendency of organic molecules from Undercooled melts. J Pharm Sci 99(9):3787-3806

Bak A, Gore A, Yanez E, Stanton M, Tufekcic S, Syed R, Akrami A, Rose M, Surapaneni S, Bostick T, King A, Neervannan S, Ostovic D, Koparkar A (2008) The co-crystal approach to improve the exposure of a water-insoluble compound: AMG 517 Sorbic acid co-crystal characterization and pharmacokinetics. J Pharm Sci 97(9):3942-3956

Bates S, Zografi G, Enger D, Morris K, Crowley K, Newman A (2006) Analysis of amorphous and nanocrystalline solid from their X-ray diffraction patterns. Pharm Res 23:2333-2349

Bevernage J, Forier T, Brouwers J, Tack J, Annaert P, Augustijns P (2011) Excipientmediated supersaturation stabilization in human intestinal fluids. Mol Pharm 8(2):564-570

Brouwers J, Brewster ME, Augustijns P (2009) Supersaturating drug delivery systems: the answer to solubility-limited oral bioavailability. J Pharm Sci 98(8): 2549-2572

Chen Y, Zhang W, Yu L (2016) Hydrogen bonding slows down surface diffusion of molecular glasses. J Phys Chem B 120(32):8007-8015

Engers D, Teng J, Jimenez-Novoa J, Gent P, Hossack S, Campbell C, Thompson J, Ivanisevic I, Templeton A, Byrn S, Newman A (2010) A solid-state approach to enable early development compounds: selection and animal bioavailability studies of an Itraconazole amorphous solid dispersion. J Pharm Sci 99(9): 3901-3922

Food and Drug Administration. Waiver of In Vivo Bioavailability and Bioequivalence Studies for Immediate-Release Solid Oral Dosage Forms Based on a Biopharmaceutics Classification System Draft Guidance, Revision 1, May 2015

Food and Drug Administration. Regulatory Classification of Pharmaceutical Cocrystals Guidance for Industry, Revision 1, August 2016, https://www.fda.gov/ downloads/Drugs/GuidanceComplianceRegulatorylnformation/Guidances/ UCM516813.pdf. Accessed 19 Apr 2017

Friesen DT, Shanker R, Crew M, Smithey DT, Curatolo WJ, Nightingale JAS (2008) Hydroxypropyl methylcellulose acetate Succinate-based spray-dried dispersions: an overview. Mol Pharm 5(6):1003-1019

Guo Y, Shalaev E, Smith S (2013) Physical stability of pharmaceutical formulations: solidstate characterization of amorphous dispersions. Trends Anal Chem 4:137-144

Hasebe M, Musemeci D, Yu L (2015) Fast surface crystallization of molecular glasses: creation of depletion zones by surface diffusion and crystallization flux. J Phys Chem B 119:3304-3311

Hussain A. Presentation on waiver of in vivo bioequivalence studies for immediate release solid oral dosage forms based on a biopharmaceutics classification system, dated November 16, 2000. Available at https://www.fda.gov/ohrms/ dockets/ac/00/slides/3657s2_01_hussain.ppt. Accessed 11 July 2017

Law D, Schmitt EA, Marsh KC, Everitt EA, Wang W, Fort JJ, Qiu Y (2004) RitonavirPEG 8000 amorphous solid dispersions: in vitro and in vivo evaluations. J Pharm Sci 93(3):563-570

Miller DP, Tan T, Nakamura J, Malcolmson RJ, Tarara TE, Weers JG (2017) Physical characterization of tobramycin inhalation powder: II. State diagram of an amorphous engineered particle formulation. Mol Pharm 14:1950-1960

Powell CT, Chen Y, Yu L (2015) Fracture of molecular glasses under tension and increasing their fracture resistance with polymer additives. J Non-Crystal Solid 429:122-128

Powell CT, Xi H, Sun Y, Gunn E, Chen Y, Ediger MD, Yu L (2015) Fast growth in oTerphenyl glasses: a possible role for fracture and surface mobility. J Phys Chem B 119(31):10124-10130

Qian KK, Bogner RH (2011) Spontaneous crystalline-to-amorphous phase transformation of organic or medicinal compounds in the presence of porous media, part 1: thermodynamics of spontaneous amorphization. J Pharm Sci 100(7):2801-2815

Qian KK, Suib SL, Bogner RH (2011) Spontaneous crystalline-to-amorphous phase transformation of organic or medicinal compounds in the presence of porous media, part 2: amorphization capacity and mechanisms of interaction. J Pharm Sci 100(11):4674-4686
Shamblin SL, Hancock BC, Pikal MJ (2006) Coupling between chemical reactivity and structural relaxation in pharmaceutical glasses. Pharm Res 23(10):2254-2268

Taylor LS, Hancock BC (2014) George Zografi and the science of solids and surfaces. J Pharm Sci 103(9):2592-2594

Taylor LS, Zhang GGZ (2016) Physical chemistry of supersaturated solutions and implications for oral absorption. Adv Drug Del Rev 101:122-142

Van Eerdenbrugh B, Baird JA, Taylor LS (2010) Crystallization tendency of active pharmaceutical ingredients following rapid solvent evaporation classification and comparison with crystallization tendency from Undercooled. Melts J Pharm Sci 99(9):3826-3838

Willart JF, Descamps M (2008) Solid state Amorphization of pharmaceuticals. Mol Pharm 5:905-920

Wunderlich B (1999) A classification of molecules, phases, and transitions as recognized by thermal analysis. Thermochim Acta 340:37-52

\section{Submit your manuscript to a SpringerOpen ${ }^{\circ}$ journal and benefit from:}

- Convenient online submission

- Rigorous peer review

- Open access: articles freely available online

- High visibility within the field

- Retaining the copyright to your article

Submit your next manuscript at springeropen.com 